\title{
Electron Density Measurements of a Linear Turbulently Heated Plasma Using a Far Infrared Interferometer
}

\author{
Kazuya SANO, Hiroto IGUCHI, Masaaki NAGATSU \\ Atsushi MASE and Takashige TSUKISHIMA
}

(Received April 21, 1982)

\begin{abstract}
Space and time resolved measurements of the electron density are made on a linear turbulently heated plasma using an optically pumped $\mathrm{CH}_{3} \mathrm{OH}$ laser interferometer. It is observed that the maximum density reaches as high as $10^{15} \mathrm{~cm}^{-3}$, and that the density decay process after the heating current has decayed, is governed by diffusion along the magnetic field.
\end{abstract}

In spite of extensive studies on turbulent heating, no clear-cut physical picture has been yet presented of the heating mechanisms. ${ }^{1)-3)}$ This is partly due to the highly nonlinear characters involved in the heating processes and partly due to difficulties in measuring fast time-varying plasma parameters. We report here the first space and time resolved measurement of the electron densities of a linear turbulent heating device at Nagoya University (THE NU-I for short), using a far infrared (FIR for short) interferometer.

THE NU-I has a pair of hollow electrodes with an inner diameter of $3 \mathrm{~cm}$, separated by $l=50 \mathrm{~cm}$ from each other. They are inserted in a glass vacuum tube having an inner diameter of $2 a=9.5 \mathrm{~cm}$ and a length of $120 \mathrm{~cm}$. A steady mirror field with a mirror ratio of 1.3 is applied. The strength of the magnetic field is $\mathrm{B}=560 \mathrm{G}$ at the mid-plain between the electrodes which are located at the mirror points. A hydrogen plasma is first introduced between the electrodes by means of a 
titanium washer-gun mounted on one end of the glass tube. The vacuum pressure before the introduction of plasma is kept on $p=2 \times 10^{-6} \mathrm{mmHg}$. A heating current $I_{\mathrm{h}}(t)$ is supplied from a $3.75 \mu \mathrm{F}$ capacitor charged to $V_{\mathrm{h}}$ which is varied from $14 \mathrm{kV}$ to $22 \mathrm{kV}$ in this experiment.

The geometrical arrangement of a Michelson-type interferometer and the plasma is schematically shown in Fig. 1. A few $\mathrm{mW}, 119 \mu \mathrm{m} \mathrm{CH}_{3} \mathrm{OH}$ line is excited in the

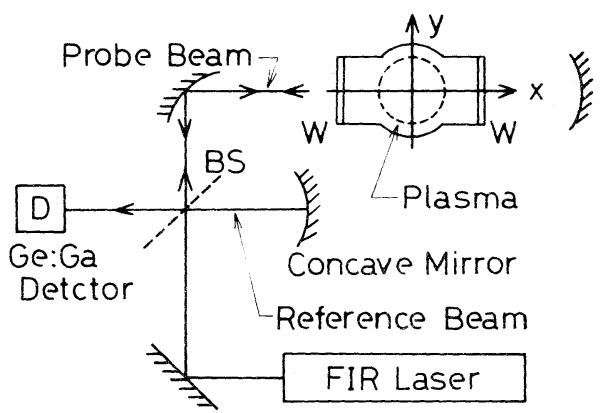

Fig. 1. Geometrical arrangement of a Michelsontype interferometer and the plasma. W: crystal quartz window,

BS : beam splitter.
FIR resonator by a pulse mode $\mathrm{CO}_{2}$ laser. The FIR beam is launched into the plasma through crystal quartz windows. The beam waist at the center of the plasma is chosen about $1 \mathrm{~cm}$ so that the beam has as small beam divergence as possible. The beam splitter consists of a maylar film of $25 \mu \mathrm{m}$ thickness. The superimposed field of the probe beam and reference beam is detected by a liquid helium-cooled Ge : Ga crystal having a sensitivity of $1.0 \mathrm{~A} / \mathrm{W}$ and a system response time less than $0.1 \mu \mathrm{s}$.

The measurements are made for two chords perpendicular to the axis of the plasma post; one passing through the axis of the plasma and the other passing through a point $2 \mathrm{~cm}$ off the axis. Examples of the chord density and heating current are shown in Fig. 2 as functions of time. The time when the heating current is set on is shown by the arrow. In Fig. 3 we plotted the maximum values of the on-axis and off-axis chord densities for various values of the heating voltage $V_{h}$. Smooth curves are drawn in Fig. 3 to obtain rough tendencies of the experimental values. The dashed curve is drawn such that its value are 0.76 times those of the solid curve.

Using Cartesian coodinates system with the origin at the center of the cross section of the cylindrical plasma and the $x$-axis directed along the FIR probe beam, 
Electron Density Measurements of a Linear Turbulently Heated Plasma Using a Far Infrared Interferometer

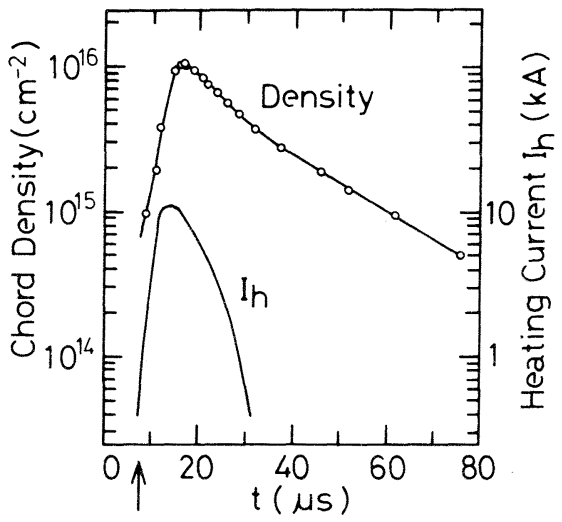

Fig. 2. Typical chord density Ystimated from the fringe numbers and heating current $I_{h}$ versus time $t$.

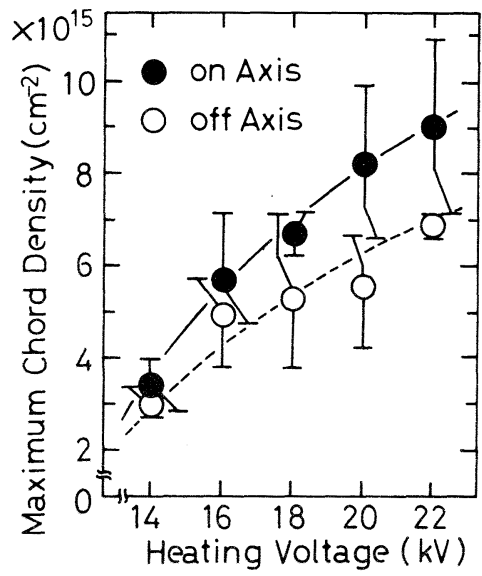

Fig. 3. Maximum values of the on-axis and $2 \mathrm{~cm}$ off-axis chord densities for various heating voltage.

the ratio of the off-axis and on-axis chord densities is given by $R(y)=\left(1-y^{2} / a^{2}\right)^{3 / 2}$, when a simple parabolic distribution of the form $n_{\mathbf{e}}(x, y)=n_{0}\left\{1-\left(x^{2}+y^{2}\right) / a^{2}\right\}$ is assumed for the electron density $n_{\mathbf{e}}(x, y)$. The experimental values of $R(y)$ for $y=2 \mathrm{~cm}$ are found to be about 0.76 for $V_{\mathrm{h}}$ 's in the range from 14 to $22 \mathrm{kV}$. Then, we have a value of $a \simeq 4.9 \mathrm{~cm}$. Figure 3 indicates that the chord density tends to saturate as $V_{\mathrm{h}}$ is increased. The value of $n_{0}$ evaluated for the parabolic distribution reaches as high as $1.0 \times 10^{15} \mathrm{~cm}^{-3}$ for $V_{\mathrm{h}}=22 \mathrm{kV}$. This indicates that the neutral hydrogen atoms or molecules with a density $n_{\mathrm{n}} \simeq 1 \times 10^{15} / \beta \mathrm{cm}^{-3}$ may exist in the glass tube, where $\beta=\alpha /(1-\alpha)$ and $\alpha$ is the degree of ionization. The corresponding particle pressure is given by $p=n_{\mathrm{n}} k T_{\mathrm{g}}=0.1 / \beta \mathrm{mmHg}$ for anassumed gas temperature of $T_{\mathrm{g}}=1000 \mathrm{~K}$, where $n_{\mathrm{n}}$ is the neutral particle density and $k$ is the Boltzmann constant. when there exist multiple ionization of impurity neutrals, the effective $\beta$-value may become large.

The density decay curve has different characters for $t<30 \mu \mathrm{s}$ and for $t>30 \mu \mathrm{s}$; 


\section{SANO $\cdot$ IGUCHI $\cdot$ NAGATSU $\cdot$ MASE $\cdot$ TSUKISHIMA}

in the latter period pure exponential decays with an e-folding time $\tau_{2}$ are observed for most shots, while in the former period the decay time $\tau_{1}$ is larger than $\tau_{2}$ for some shots. On the average, however, $\tau_{1}$ is less than $\tau_{2}$, and we obtained a shotsavaraged value of $\tau_{2} \simeq 20.0 \pm 3.5 \mu \mathrm{s}$. It is interesting to note that the heating current exists only during the first period $(t<30 \mu \mathrm{s})$. Then, a slight electron heating or ionization may take place until $t \simeq 30 \mu \mathrm{s}$. On the other hand, for $t>30 \mu \mathrm{s}$ the plasma is thought as a kind of afterglow where the electrons have been cooled dow $n$ to, say, $T_{\mathrm{e}} \simeq 10 \mathrm{eV}$. The exponential decay of $n_{\mathrm{e}}$ in later period suggests that it is controlled by diffusion process. Since the Debye length $\lambda_{D}$ has a value of $2.4 \times 10^{-4} \mathrm{~cm}$ for $T_{\mathrm{e}} \simeq 10 \mathrm{eV}$ and $n_{\mathrm{e}} \simeq 10^{14} \mathrm{~cm}^{-3}$, which is much smaller than the plasma radius $a \simeq 5 \mathrm{~cm}$, an ambipolar diffusion along and across the magnetic field may dominate. In order to obtain an order-of-magnitude estimate of the e-folding decay time, We have made numerical evaluations of diffusion coefficients along and across the magnetic field, and the corresponding decay times. The results are tabulated in Table-I, where $m$ and $M$ are the masses of an electron and an ion, respectively, and $\nu_{\mathrm{em}}$ and $\nu_{\mathrm{in}}$ are the collision freguencies of an electron and ion with neutrals. The value of $\nu_{\mathrm{em}}$ and $\nu_{\mathrm{im}}$ are evaluated from the relations, $\nu_{\mathrm{em}}=p P_{\mathrm{ce}} \sqrt{k T_{\mathrm{e}} / m}=$ $1.2 \times 10^{8} / \beta \mathrm{s}^{-1}$ and $\nu_{\mathrm{im}}=p P_{\mathrm{ci}} \sqrt{k T_{\mathrm{i}} / M}=1.75 \times 10^{6} / \beta \mathrm{s}^{-1}$ with $P_{\mathrm{ce}}=40 \mathrm{~cm}^{-1} \mathrm{mmHg}^{-1}$ and $P_{\mathrm{ci}} \simeq 200 \mathrm{~cm}^{-1} \mathrm{mmHg}^{-1}$ for $T_{\mathrm{e}}=10 \mathrm{eV}$ and $T_{\mathrm{i}}=0.1 \mathrm{eV}$, respectively. ${ }^{4)}$

Table I. Diffusion coefficients and the e-folding decay time

\begin{tabular}{c|cc}
\hline & $\mathrm{D}_{\mathrm{a}}\left(\mathrm{cm}^{2} / \mathrm{s}\right)$ & $\tau(\mu \mathrm{s})$ \\
\hline Along $B$ & $D_{\mathrm{a} \| 1}=\frac{k T_{\mathrm{e}}}{M \nu_{\mathrm{im}}} \simeq 5.5 \times 10^{6}$ & $\tau_{1:}=\left(\frac{\ell}{\pi}\right)^{2} \frac{1}{D_{\mathrm{a}}} \simeq \frac{46}{\beta}$ \\
$\operatorname{Across} B$ & $D_{\mathrm{a} i} \simeq \frac{k T_{\mathrm{e}}}{m} \cdot \frac{\nu_{\mathrm{e}}}{\omega_{\mathrm{ce}}^{2}} \simeq 1.8 \times 10^{4}$ & $\tau_{\perp}=\left(\frac{\mathrm{a}}{2.54}\right)^{2} \frac{1}{D_{\mathrm{a}}} \simeq 142 \beta$ \\
\hline
\end{tabular}




\section{Electron Density Measurements of a Linear. Turbulently Heated}

Plasma Using a Far Infrared Interferometer

The numerical values shown in Table-I suggest that the plasma is lost primarily by diffusion along the magnetic field. The $\mathrm{e}=$ folding time $\tau_{\| 1}$ roughly coincides with the experimental one, provided $T_{\mathrm{e}} \beta=20 \mathrm{eV}$. For $\beta=2$, we have $T_{\mathrm{e}}=10 \mathrm{eV}$ and $p=0.05 \mathrm{mmHg}$. The relatively high value of $p$ may have been brought forth partly by the recombinations of the initial high density plasma and partly by the evaporations from the titanium washer-gun. The neutral particles so introduced are pumped out with an e-folding time of $11 \mathrm{~ms}$ in the present apparatus. Thus, the neutral pressure remains virtually unchanged during the decay process of the electron density. It is finally added that, in spite of the increase of the neutral particles, the classical resistivity still remains less than the enchanced resistivity observed in early time of the heating process.

The present work is supported in part by Grant-in-Aid for Scientific Reserch from the Ministry of Education, Science and Culture.

\section{References}

1) H. de Kluiver : Rijnhuizen Rep. 77-106 (1976) Associatie Euratom-FOM.

2) H. Iguchi : J. Phys. Soc. Jpn. 45 (1978) 1364.

3) O. Asada, K. Yoshioka, A. Inoue and T. Tsukishima : Jpn. J. Appl. Phys. 20 (1981) 173.

4) S. C. Brown : Basic Data of Plasma Physics (John Wiley \& Sons, New York, $1959)$ p.5, p. 33. 